\title{
Electron Microscopy Study of Porous and Co Functionalized BaTiO3 Thin Films
}

\author{
P. Ferreira*, A. Castro*, P. M. Vilarinho*, M. -G. Willinger*, J. Mosa**, C. Laberty** and C. \\ Sanchez** \\ * Department of Ceramics and Glass Engineering, CICECO, University of Aveiro, Campus de \\ Santiago, Portugal, 3810-193 Aveiro \\ ** LCMCP, UMR-7574 CNRS, UPMC, Collège de France, Université Paris 06, 11 Place Marcelin \\ Berthelot, France, 75231 Paris
}

Multiferroics are currently of great interest for applications in microelectronics namely in future data storage and spintronic devices. These materials couple simultaneously ferroelectric and ferromagnetic properties and have potentially different applications resulting from the coupling between their dual order parameters. A true multiferroic material is single phase. However, the known true multiferroic materials possess insufficient coupling between the two phenomena or their magnetoelectric response occurs at temperatures too low to be useful in practical applications. But a tremendous progress in the field of microelectronics can be expected if one is able to design an effective multiferroic material with ideal coupling of the ferromagnetic and ferroelectric properties to suit a particular application [1]. Within this context composite structures are gaining considerable interest and different strategies in terms of materials microstructure have been proposed including horizontal multilayers and vertical heterostructures. In the horizontal multilayer heterostructures, the alternating layers of conventional ferro/ferrimagnetic and ferroelectric phases are grown, while in the vertical heterostructures nanopillars of the ferro/ferrimagnetic phase are embedded in a ferroelectric matrix. The later structures show advantages over the first ones because promote larger interfacial surface area and are intrinsically heteroepitaxial in three dimensions; which is expected to allow a stronger coupling between ferroelectric and ferromagnetic components [1].

In this work, we report a systematic microstructural analysis of ferroic composite films in which a porous ferroelectric matrix is impregnated with a magnetic metal. Porous ferroelectric barium titanate thin films were prepared using a sol-gel process based on evaporation induced self-assembly of blockcopolymer to direct the formation of the pore arrays of ferroelectric phase on conductive substrates $(\mathrm{Pt} / \mathrm{TiO} 2 / \mathrm{SiO} 2 / \mathrm{Si})$ [2]. By electrochemical deposition, the pores of the ferroelectric films were filled with cobalt metallic nanoparticles forming the composite materials.

The porous ferroelectric thin films were thermally treated at different temperatures in order to establish the relation between ordering and microstructure crystallization before functionalization with magnetic nanoparticles. High Resolution Transmission Electron Microscopy (HRTEM) was one of the techniques used to do this study (Figure 1). The microstructure of the films is porous and the porosity is kept after heat treatment up to $750{ }^{\circ} \mathrm{C}$. However the organization of the pores is degraded upon thermal treatment due to films crystallization. At $625^{\circ} \mathrm{C}$ the microstructure is pseudo-amorphous, presenting only a few crystalline domains, as proved by Selected Area Electron Diffraction (SAED) micrographs. Nevertheless, as the annealing temperature increases $\left(700\right.$ and $\left.750^{\circ} \mathrm{C}\right)$ the crystalline phase becomes more evident leading to the appearance of defined ring patterns in the SAED micrographs. In addition, Scanning Electron Microscopy (SEM) was used to assess films microstructure and to evaluate films degree of ordering before and after functionalization of ferroelectric films thermally treated at $650^{\circ} \mathrm{C}$ with Co nanoparticles (Figure 2).

It is clear that the porous matrix of $\mathrm{BaTiO} 3$ can be functionalized with Co nanoparticles by electrochemical deposition technique (Figure $2 \mathrm{a}$ and $2 \mathrm{~b}$ ).

The thermal treatment of the composite film at $700{ }^{\circ} \mathrm{C}$ seems to improve the interfaces as shown in Figure 2c. Further studies on the ferroelectric and ferromagnetic properties are need to evaluate the multiferroic characteristics of the composites. 


\section{References}

1. R. Ramesh, N. A. Spaldin, Nature Mater. 6: 21, 2007

2. P. Ferreira, R. Z. Hou, A. Wu, M.-G. Willinger, P. M. Vilarinho, J. Mosa, C. Laberty-Robert, C. Boissière, D. Grosso, C. Sanchez, Langmuir (accepted)

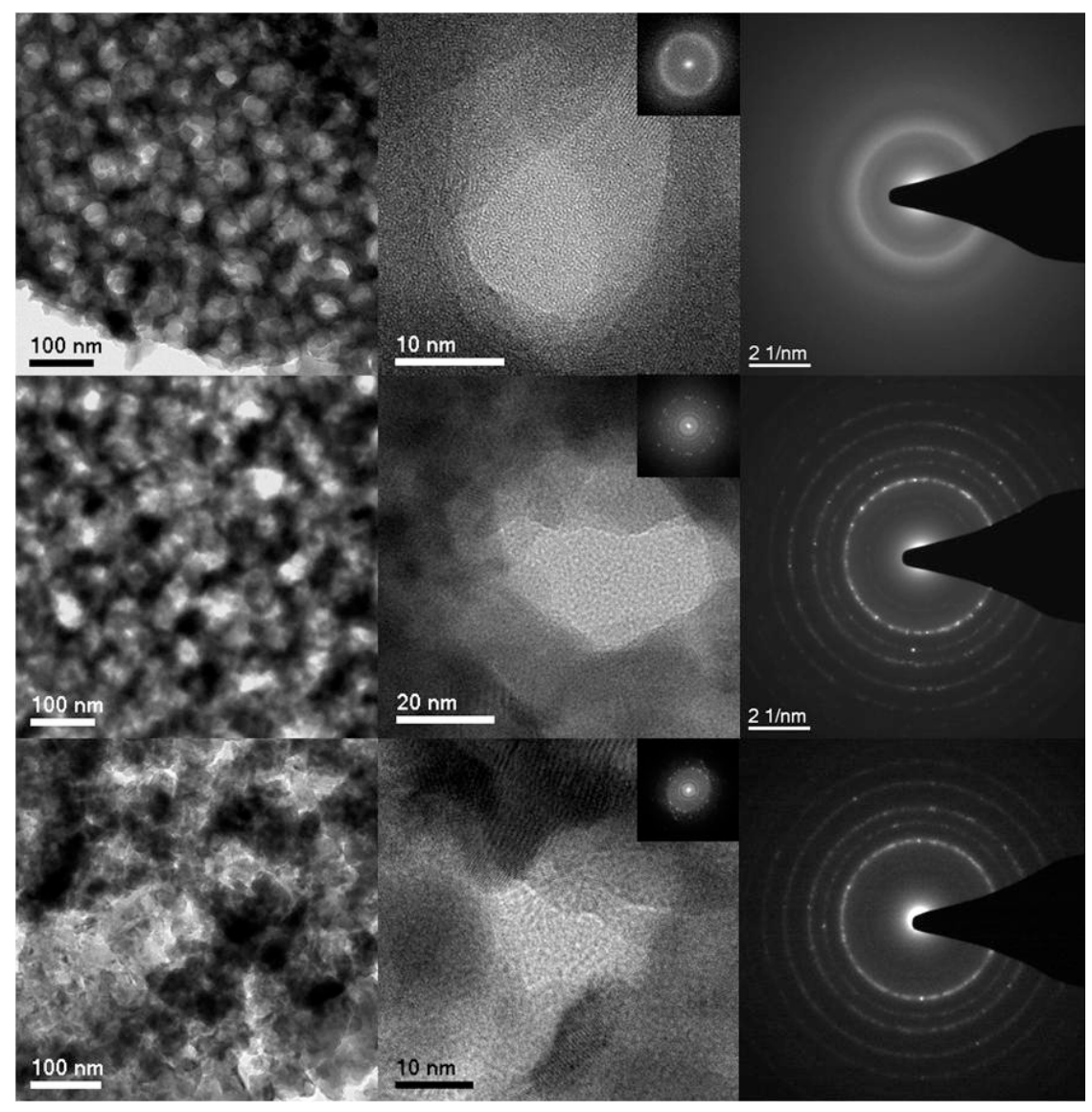

Figure 1. TEM, HRTEM and SAED micrographs of porous BaTiO3 thin films after thermal treatment at: a) $625{ }^{\circ} \mathrm{C}$, b) $700{ }^{\circ} \mathrm{C}$ and c) $750{ }^{\circ} \mathrm{C}$. Insets in the central column show the corresponding power spectrum and demonstrate the presence of lattice fringes in the micrographs.

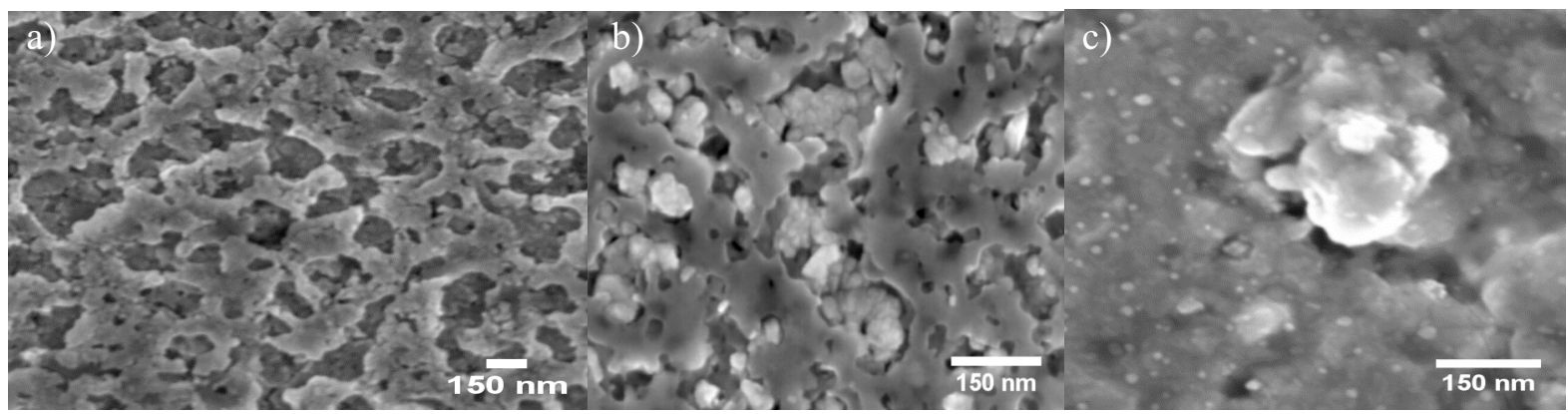

Figure 2. SEM micrographs: a) porous $\mathrm{BaTiO} 3$ thin films thermally treated at $650^{\circ} \mathrm{C}, \mathrm{b}$ ) the same film after functionalization with Co nanoparticles and c) a composite films treated at $700^{\circ} \mathrm{C}$.

Acknowledgment: The authors are grateful to FCT and FEDER (QREN - COMPETE) for financial support (PTDC/CTM/098130/2008). 